\section{MICROBIAL POPULATIONS}

Evolutionary relationships and the diversity of the rumen bacteria belonging to the CytophagaFlexibacter-Bacteroides phylum. G Avguštin', A Ramšak', M Peterka', FV Nekrep', HJ Flint ${ }^{2}$ 'University of Ljubljana, BF, Zootechnical Department, Groblje 3, 1230 Domzale, Slovenia; ${ }^{2}$ Rowett Research Institute, Bucksburn, Aberdeen, AB2 9SB, UK)

In the last decade three Bacteroides species found in the rumen, $B$. ruminicola, $B$. succinogenes and $B$. amylophilus, have all been transferred to the newly created genera, Prevotella, Fibrobacter and Ruminobacter, respectively. $P$. ruminicola has since been shown to comprise genotypically heterogeneous strains and reclassification into four new species, $P$. ruminicola, $P$. bryantii, P. brevis and P. albensis, is proposed $[1,2]$. Our understanding of diversity among rumen Prevotella is currently limited to available cultured isolates, however, and it is important to establish whether additional diversity exists that has not been cultivated, and whether certain groups have been underestimated due to cultural bias.

PCR amplification of ribosomal DNA sequences from environmental samples, followed by cloning and sequencing, has allowed examination of the diversity present in complex microbial ecosystems such as hydrothermal vents, sea sediments and soil $[3,4]$. Here total DNA was extracted from a liquid rumen sample from a black and white Friesian cow, and the 16S rRNA genes amplified in a PCR reaction. Conserved bacterial primers with added restriction sites for the NotI and SalI endonucleases were used for the amplification of approximately $1400 \mathrm{bp}$ of rDNA. The amplification products were ligated in a plasmid vector and electroporated into Escherichia coli recipient cells.

Over 650 clones carrying a 16S rRNA gene copy were recovered and hybridised to a range of broad and species-specific oligonucleotide probes. Approximately $10 \%$ of the clones hybridised to one of two different probes (BacPre and CFB$\mathrm{CF}$ ) recognising organisms from the Cytophaga-Flexibacter-Bacteroides phylum, while none of the clones hybridised to a third probe CFB-B specific for the Bacteroides cluster of the CFB phylum [4]. None of the clones hybridised to probes specific for Fibrobacter succinogenes. Ruminococcus flovefaciens and $R$. albus, all known as prominent cellulolytic rumen bacteria. Restriction analysis of the amplified genes recognised by the BacPre probe [1] was conducted using TaqI, $D d e \mathrm{I}, A l u \mathrm{I}$ and $H h a \mathrm{I}$ endonucleases. This demonstrated a considerable number of $16 \mathrm{~S}$ rRNA profiles suggesting that a considerable range of species belonging to the CFB phylum inhabits the rumen.

Approximately 500 bases of $16 \mathrm{~S}$ rDNA were sequenced from 18 of the BacPre group of clones. Of these seven showed a close relationship $(90-98 \%$ sequence similarity) with $P$. ruminicola $23^{\mathrm{T}}$ whilefive were most closely related to $P$. ru minicola $\mathrm{Tc2}-24$ and another five were most closely related to $P$. ruminicola $23^{\mathrm{T}}$ but showed only a low level of similarity (77-90\%). No two sequences were the same. These results affirm the abundance of $P$. ruminicola-related bacteria in the rumen, independent of cultural bias, but also emphasise the enormous diversity 


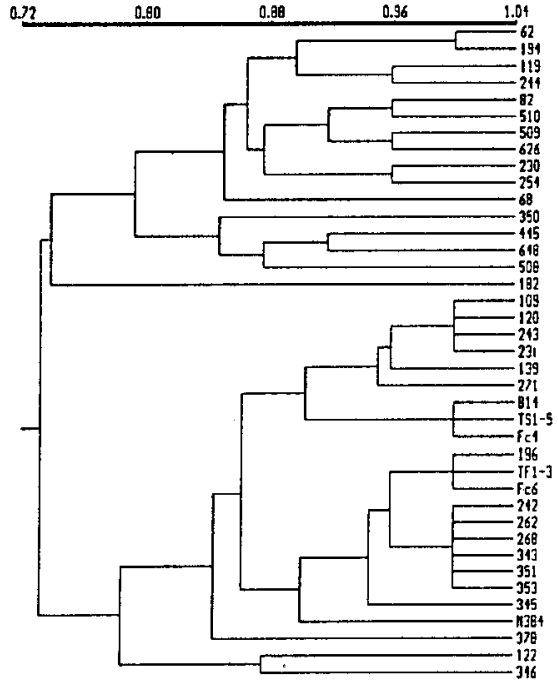

Dendrogram based on restriction enzyme cleavage patterns obtained with TaqI, HhaI, $A l u \mathrm{I}$ and DdeI for PCR-amplified 16SrDNA from clones recognised by the BacPre probe. An unweighted pair group method analysis (UPGMA) was performed. Profiles from cultured Prevotella strains $\mathrm{B}_{1} 4, \mathrm{M} 384,23$, TF13 , TS1-5, FC2, FC4) are included for comparison.

of Prevotella strains present in a single animal.

1. Avguštin G, Wright F, Flint HJ (1994) Int $J$ Syst Bacteriol 44, 246-255

2. Avguštin G, Wallace RJ, Flint HJ (1997) Int J Syst Bacteriol 47, 284-288

3. Amman RI, Krumholz L, Stahl DA (1990) $J$ Bacteriol 172, 762-770

4. Amman RI, Ludwig W, Schleifer K-H (1995) Microbiol Rev 59, 143-169

An investigation of microbial diversity in the rumen of dairy cattle using comparative sequence analysis of cloned 16S rRNA genes. RJ Forster, MF Whitford, CE Beard, J Gong (Centre for Food and Animal Research, Agriculture and Agri-Food Canada, Ottowa, ON; Canada, KIA OC6)

The concentration of bacterial cells in the

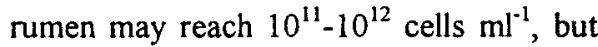
it is usually possible to culture only a small fraction of these numbers. Bacteria in the rumen have generally been studied after isolation on selective media. Bacteria which are not able to grow on selective or non-selective media may include cells of known types that are inviable or unable to reproduce. Bacteriocins, which have been shown to be prevalent amongst the genus Butyrivibrio [1], would also inhibit the growth of many strains in vitro. However it may also be that most in vitro culture conditions are simply not able to support the growth of many rumen bacteria. The extent to which unfamiliar bacterial strains contribute to the rumen ecosystem is therefore unknown. This is of concern to rumen microbiologists, especially when effects of novel or genetically engineered rumen bacteria on the rumen ecosystem need to be evaluated.

In preliminary attempts to address this problem we have directly amplified $16 \mathrm{~S}$ rRNA gene sequences from ruminal fluid samples of dairy cattle. Total DNA was extracted from the rumen fluid of ten cattle fed haylage/corn silage/concentrate rations at two different times, using a beadbeating, phenol/chloroform extraction method. Primers which are homologous to most 16S rRNA genes were used in PCR reactions to amplify almost complete $16 \mathrm{~S}$ rRNA gene sequences (approx. $1450 \mathrm{bp}$ ). One set of DNA extractions was amplified using either 12 or 30 cycles of PCR in order to examine biases introduced during 